Rev. salud pública. 10 (4):583-592, 2008

\title{
Barreras Geográficas y Económicas para el Acceso a los Servicios Oncológicos del Instituto Nacional de Cancerología en Bogotá
}

\author{
Geographical and economic barriers to access to Oncology services \\ offered by the National Cancer Institute in Bogota, Colombia \\ Juan Carlos García-Ubaque ${ }^{1}$ y Carmen S. Quintero-Matallana² \\ 1 Facultad de Enfermería. Universidad Nacional de Colombia, Bogotá D.C. jcgarciau@unal.edu.co \\ 2 Escuela de Administración Pública-ESAP; Instituto Nacional de Cancerología-E.S.E., Bogotá, \\ D.C. Colombia cquintero@incancerologia.gov.co
}

Recibido 20 Febrero 2008/Enviado para Modificación 20 Agosto 2008/Aceptado 3 Septiembre 2008

\section{RESUMEN}

Objetivo Se realiza una aproximación a las barreras geográficas y económicas para el acceso a los servicios oncológicos del Instituto Nacional de CancerologíaINC; Se presenta la necesidad de plantear alternativas para afrontar estas problemáticas y considerar estudios que evalúen problemáticas similares y sus soluciones

Métodos Se utilizaron los métodos cualitativo y cuantitativo en lo referente a datos estadísticos, dentro de un contexto de interés social, con un enfoque empírico analítico.

Resultados Se encontró que durante el 2005, tuvieron acceso al programa de albergues solo 176 pacientes de los 3391 pacientes provenientes de regiones del país distintas a Bogotá atendidos en el mismo periodo; es decir que el programa solo cubrió el 5,19\% de los pacientes de diferentes lugares del país. De igual manera se encontró que se debe afrontar la inequidad de género que hasta la fecha se ha venido presentando con los varones.

Conclusiones Se deben buscar alternativas de alojamiento diferentes a los albergues, que hagan posible el acceso al tratamiento especializado con dignidad y calidad de vida. El estudio permitió plantear alternativas de solución a corto, mediano y largo plazo.

Palabras Clave: Equidad en salud, accesibilidad a los servicios de salud, cáncer, atención hospitalaria (fuente: DeCS, BIREME).

\section{ABSTRACT}

Objective Geographical and economic barriers to access to National Cancer Institute $(\mathrm{NCl})$ oncology services were evaluated; such approach revealed the need for 
alternatives for dealing with these issues and considering studies aimed at assessing similar problems and resolving them.

Methods An analytical empirical approach used qualitative and quantitative statistical methods were used within a context of social concern.

Results Only 176 out of 3391 patients had access to the "Temporary-housing programme" during 2005 , covering $5,19 \%$ of all patients coming from remote regions of Colombia. Most patients were women who were looking for alternatives outside the institutional programmes being offered.

Conclusion There is a need to fix gender inequity and search for better housing alternatives to providing refuges, providing access to specialised treatment and the need for attending people with dignity and ensuring quality of life. The study led to proposing new short-, medium- and long-term alternatives for improving health service access.

Key Words: Social justice, health service accessibility, cancer care facility, hospital care, refuge (source: MeSH, NLM).

n Colombia el Sistema de Protección Social se constituye en el conjunto
de políticas públicas orientadas a disminuir la vulnerabilidad y a mejorar
la calidad de vida de los colombianos, especialmente de los más desprotegidos. Este enfoque implica la identificación y estudio de los riesgos y la definición de estrategias que faciliten a la población enfrentar los mismos. En este campo el tema de la salud resulta crucial (1-3) y, por tanto es importante considerar lo atinente al acceso a los servicios de salud por parte de las poblaciones más vulnerables.

Por otra parte, Wiesner et al (4) insisten en que el cáncer es un problema importante de salud pública en Colombia, debido a que las tasas de incidencia y de mortalidad se han venido incrementando en las últimas décadas. Esta situación ha generado un grave impacto social y económico para el país si se tiene en cuenta que cerca de 28 mil hombres y mujeres mueren anualmente por esta causa y que la mayor parte de los casos se diagnostican cuando la enfermedad está muy avanzada y las posibilidades para que la terapia surta efecto son muy limitadas. Plazas et al (5) destacan que el cáncer, como enfermedad crónica, es uno de los principales problemas de salud después de las enfermedades cardiovasculares, con base en las cifras de la Organización Mundial de la Salud (OMS) en las que resalta que esta patología es la segunda causa de muerte en los países industrializados y fue la tercera causa de muerte en Colombia, en el año 2002. Plantea que siendo el cáncer una condición tan frecuente y mostrando la tendencia al incremento ya descrita, es de extraor- 
dinaria importancia evaluar las condiciones en las que se prestan los servicios a estos pacientes y las oportunidades de atención a los mismos.

Por ser el tratamiento del cáncer de muy alta complejidad y costo, no se justifica llevar servicios de atención específica a cada municipio del país; por tanto, se deben traer los pacientes que padecen cáncer a centros de referencia, que cuenten con la infraestructura y la experticia necesarias para ofrecer la mejor alternativa de tratamiento. En Colombia este centro es el Instituto Nacional de Cancerología-INC, la institución más importante del país por experiencia y reconocimiento en este campo. Ahora bien, el traslado desde diferentes lugares del país implica diversos grados de problemas económicos y de acceso, los cuales no corresponden exclusivamente al pago de los servicios asistenciales, sino que a esto se le suma el transporte, los problemas de permanencia y manutención en una gran ciudad y el no tener con frecuencia acceso a un alojamiento en el que se les brinde apoyo para ubicarse y desplazarse en la ciudad, por lo que es posible que muchos de estos pacientes desistan de recibir el tratamiento especializado que necesitan. Por otra parte, el aparato judicial, a través de sus diferentes jurisdicciones e instancias y por vía de sentencias judiciales de tutela, ha compensado o cubierto muchos de los eventos relacionados con el tratamiento del cáncer dentro del Sistema General de Seguridad Social en Salud Colombiano (6).

En este aspecto es claro que no existe el conocimiento suficiente acerca de cómo reciben los servicios del INC quienes proceden de lugares diferentes a Bogotá. Por tanto, se presenta una aproximación a las barreras geográficas y económicas para el acceso a los servicios oncológicos del Instituto Nacional de Cancerología INC, buscando describir la situación actual y examinar algunas alternativas orientadas a minimizar los efectos de estas situaciones en el tratamiento de esta patología.

\section{MÉTODOS}

Se efectuó un estudio descriptivo de carácter exploratorio. Se utilizaron métodos cualitativos y cuantitativos, dentro de un contexto de interés social, como parte de un enfoque empírico analítico. Se utilizaron técnicas de revisión bibliográfica y de fuentes documentales, observaciones de campo, consulta con expertos, encuestas a usuarios y técnicas de análisis de información. En primera instancia y para aproximarse a la magnitud del problema, se procedió a establecer el perfil de los pacientes del Instituto; se consultaron las bases de datos de consulta externa extractando lo relacionado con las 
variables de afiliación, procedencia, género y escolaridad, entre otros. Estos datos fueron procesados utilizando una base de datos Excel ${ }^{\circledR}$. Una vez establecido este perfil se procedió a conocer el programa de albergues del Instituto, respuesta que actualmente se brinda por parte del área de Trabajo Social del INC para la población procedente de zonas diferentes a Bogotá. Se procedió a entrevistar a las personas responsables del programa y a verificar los informes de gestión existentes al respecto. Para obtener información adicional a la del INC, se aplicó una encuesta a una muestra aleatoria de 50 pacientes procedentes de diferentes ciudades del país que estaban recibiendo quimioterapia, radioterapia o estaban en trámites prequirúrgicos. Se les preguntó acerca de las implicaciones que tenía el desplazamiento a la capital para recibir los servicios de atención a su enfermedad y como estaban resolviendo el problema de alojamiento y manutención. La información derivada de esta encuesta fue procesada utilizando una base de datos Excel ${ }^{\circledR}$.

Posteriormente se efectúo un análisis financiero de las implicaciones de esta problemática, en primera instancia revisando los costos en los que incurre el Instituto para el sostenimiento del albergue que ofrece y, en segunda instancia, indagando acerca del gasto directo en el que incurren los pacientes cada vez que se desplazan a Bogotá para recibir el servicio. Se solicitó información acerca de los costos que implica tanto el desplazamiento, como el alojamiento y la manutención. Finalmente se analizo esta información y compartió con expertos con el fin de conocer sus percepciones y recomendaciones acerca de cómo se puede afrontar esta problemática.

\section{RESULTADOS}

De los pacientes atendidos en el año 2005 en el Instituto, el 55,1 \% pertenece a la población definida como pobre y vulnerable (subsidiado o vinculados), pero aunque el 33,4\% de los pacientes pertenece al régimen contributivo, es importante señalar que dentro de este rango se ubican personas con ingresos inferiores a dos salarios mínimos; Es decir que en su mayoría los pacientes del Instituto son personas de bajos recursos, que eventualmente necesitan apoyo para acceder a la atención y al tratamiento. Respecto al indicador de procedencia, el $47 \%$ de los pacientes proviene de fuera de Bogotá, siendo en su mayoría de la región Andina (72 \%), lo que nos cuestiona acerca del acceso efectivo de los pacientes de lugares apartados del país, quienes pueden verse marginados de la tecnología y el conocimiento científico. El 11 \% 
son iletrados y el $55 \%$ solo tiene estudios de primaria-muchos incompleta, lo que sumado a la procedencia de diversas regiones puede generar un choque cultural fuerte y una permanencia difícil en la ciudad.

Al revisar el programa de albergues del Instituto, se encontró que se dispone de posibilidad para niños solos o con acompañante y para mujeres con acompañante; Es decir que no se dispone de albergues para varones lo que puede evidenciar una inequidad de género si se tiene en cuenta que el 37\% de las atenciones del periodo fueron brindadas a varones. Se encontró también que en el año 2005 tuvieron acceso al programa de albergues del INC 176 pacientes de los 3391 pacientes atendidos en la misma vigencia provenientes de regiones del país diferentes a Bogotá, es decir que el programa solo cubrió el 5,2 \% de los usuarios potenciales.

$\mathrm{Al}$ aplicar la encuesta se halló que un gran porcentaje de los pacientes que son atendidos en el INC, provenientes de diferentes regiones de todo el país, se hospedan en hogares de familiares o amigos y cuando no tienen ningún contacto o apoyo en la ciudad recurren a hoteles o a alquilar lugares amoblados o habitaciones en casas de familia. Estos lugares muy pocas veces resultan apropiados, ya que adolecen de condiciones para movilizar a pacientes con algún grado de discapacidad, implican largos trayectos en transporte urbano de baja calidad y en términos generales representan una alteración del ritmo de vida de la familia receptora que es percibida por el paciente con sensación de culpa. Adicionalmente, los resultados de la encuesta alertaron acerca del gasto de bolsillo en el que estaban incurriendo los pacientes y sus familias para asistir al tratamiento.

Al efectuar la revisión de los costos, encontramos que el costo del albergue de los pacientes del INC en el año 2005 fue de \$ 91792 000, monto asumido por el presupuesto del Instituto, donaciones y el cargo que se realiza a los pacientes. El perfil de costos indirectos de la atención se presenta en las siguientes Tablas.

Como se observa, los costos de alojamiento en hotel son bastante altos pues oscilan entre \$2 485000 y \$ 21655000 (para el compuesto paciente-acompañante), dependiendo del tiempo de permanencia. Pero adicional a esto se deben cubrir otros gastos, como el transporte interdepartamental para desplazarse desde su lugar de origen hasta Bogotá (Tabla 2). 
Tabla 1. Costos de alojamiento para el promedio de días de tratamiento de un paciente y su acompañante al año

\begin{tabular}{|c|c|c|c|c|c|c|}
\hline Tipo de alojamiento & $\begin{array}{c}\text { Variación } \\
\text { de costo } \\
\$(000)\end{array}$ & $\begin{array}{l}\text { Costo } \\
\text { minimo } \\
\text { noche } \\
\text { individual } \\
\$(000)\end{array}$ & $\begin{array}{c}\text { Costo minimo } \\
\text { noche } \\
\text { habitación } \\
\text { doble } \\
\$(000)\end{array}$ & $\begin{array}{l}\text { Promedio } \\
\text { dias de } \\
\text { estancia } \\
\text { al an̆o }\end{array}$ & $\begin{array}{l}\text { Valor total } \\
\text { para el } \\
\text { paciente } \\
\$(000)\end{array}$ & $\begin{array}{c}\text { Valor total } \\
\text { (paciente- } \\
\text { acompañante) } \\
\$(000)\end{array}$ \\
\hline $\begin{array}{l}\text { Hoteles económicos } \\
\text { centro de Bogoté }\end{array}$ & $30-45$ & 30 & 35 & 71 & 2130 & 2485 \\
\hline Hotel del Sena & $53-74$ & 53 & 74 & 71 & 3763 & 5254 \\
\hline Hostales & $45-90$ & 45 & 66 & 71 & 3195 & 4686 \\
\hline Apartahoteles & $80-140$ & 80 & 80 & 71 & 5680 & 5680 \\
\hline Hoteles intermedios & $80-125$ & 80 & 125 & 71 & 5680 & 8875 \\
\hline $\begin{array}{l}\text { Hoteles } 5 \text { estrellas } \\
\text { (habitación estándar) }\end{array}$ & $275-310$ & 275 & 305 & 71 & 9525 & 21655 \\
\hline
\end{tabular}

Fuente: Costos en pesos Colombianos determinados telefónicamente en el mes de octubre de 2006, para referencia el Salario. Mínimo mensual de 2006 en Colombia fue de \$408 000,00

Tabla 2. Costos de transporte interdepartamental para el promedio de estancias de un paciente y su acompañante al año

\begin{tabular}{|c|c|c|c|c|c|}
\hline $\begin{array}{l}\text { Tipo de } \\
\text { Transporte }\end{array}$ & $\begin{array}{c}\text { Variación } \\
\text { Costo } \\
\text { Interdepartamental } \\
\$(000)\end{array}$ & $\begin{array}{c}\text { Costo } \\
\text { Promedio } \\
\$(000)\end{array}$ & $\begin{array}{l}\text { Promedio } \\
\text { Estancias } \\
\text { Año }\end{array}$ & $\begin{array}{l}\text { Valor Total } \\
\text { Promedio } \\
\text { Anfo } \\
\text { (Paciente) } \\
\text { S(000) }\end{array}$ & $\begin{array}{l}\text { Valor Total } \\
\text { Promedio } \\
\text { (Paciente- } \\
\text { Acompañante) } \\
\text { S(000) }\end{array}$ \\
\hline Aẹreo & $380-670$ & 525 & 6 & 6300 & 12600 \\
\hline Terrestre & $5-206$ & 105,5 & 6 & 1266 & 2532 \\
\hline
\end{tabular}

Este traslado, dependiendo de muchos factores como el estado de salud del paciente, puede ser aéreo o terrestre, igualmente debe considerarse que por lo general se demanda que se asista con acompañante, así que se debe disponer de dinero adicional para cubrir el traslado de este familiar.

Además de los costos de alojamiento y de traslado interdepartamental están los gastos de alimentación y de transporte urbano Tabla 3.

Revisando las Tablas precedentes se observa que si el paciente no tiene o no utiliza los hogares de familiares o amigos en Bogotá para hospedarse y si no accede al programa de albergues, debe disponer de unos recursos mínimos calculados para 6 estancias al año, así:

Paciente solo \$ 5831300 (traslado terrestre interdepartamental).

- $\quad$ Paciente-acompañante $\$ 9073000$ (traslado terrestre interdepartamental para dos personas). 
Tabla 3. Otros costos para el paciente y su acompañante durante los días

\begin{tabular}{|c|c|c|c|c|c|}
\hline Tipo de gasto & $\begin{array}{c}\text { Oscilación } \\
\text { de costos } \\
\$(000)\end{array}$ & $\begin{array}{c}\text { Costo } \\
\text { promedio } \\
\$(000)\end{array}$ & $\begin{array}{l}\text { Promedio } \\
\text { dias de } \\
\text { estancia al } \\
\text { an̆o }\end{array}$ & $\begin{array}{c}\text { Valor total } \\
\text { promedio } \\
\text { (Paciente) } \\
\$(000)\end{array}$ & $\begin{array}{c}\text { Valor total } \\
\text { promedio } \\
\text { (paciente- } \\
\text { acompañante) } \\
\$(000)\end{array}$ \\
\hline Alimentación & $7,5-10,5$ & 9 & 71 & 1278 & 2556 \\
\hline $\begin{array}{l}\text { Transporte en la } \\
\text { ciudad de Bogotá }\end{array}$ & $1,3-15$ & 8,15 & 71 & 1157,3 & 1500 \\
\hline
\end{tabular}

\section{DISCUSIÓN}

A pesar de la postura de autores como Jaramillo (7), como lo señala Hernández (8), la equidad del sistema de salud Colombiano ha sido ampliamente debatida (9-14), llegándose a la conclusión de que el actual modelo evidencia la idea de la salud como un servicio de consumo individual, el cual puede universalizarse por la vía del mercado y por tanto se llega a ocultar los problemas de equidad acumulados y ligados a la capacidad de pago de las personas. No obstante, este problema no es exclusivo del sistema de salud Colombiano, tal y como se evidencia en la investigación de Regidor (15), quien cita estudios de Drever (16), Mackenbach (17) y Kunst (18), entre otros. Es de destacar que autores como Mejía-Mejía (19) insisten en el impacto de la ubicación geográfica en los problemas relacionados con el acceso a los servicios, lo cual es corroborado por Ruiz-Rodríguez (20) en relación con la atención de la población de desplazados por la violencia en una región Colombiana.

La investigación acerca de los problemas de acceso, relacionados con la atención de padecimientos específicos, en particular el cáncer, no tiene un amplio desarrollo. Sin embargo, hay estudios importantes como el de Modeste et al (21), en el que, con respecto a la mamografía, se destacan las barreras geográficas y económicas que afrontan las habitantes de Tobago para acceder al servicio, lo cual fue ratificado por Angus (22) en Canadá. En el estudio de Fonseca et al (23) se encuentra satisfacción de los pacientes oncológicos por la atención brindada en los servicios ambulatorios, pero se destacan los problemas de accesibilidad. En el estudio realizado por Restrepo et al (24) se señala que existen barreras de tipo económico o geográfico para acceder al servicio de la citología vaginal, por dificultades para llegar al sitio de atención y por tener que asumir costos de transporte elevados y tiempos de espera, entre otros, lo cual es corroborado por el trabajo de Palacio-Mejía (25), en el 
que se encuentra la influencia de las inequidades en la mortalidad por cáncer cervicouterino en México y por Lucumi y Gómez (26), que resalta la inequidad que produce la marginalidad en la realización de actividades de diagnóstico precoz. Finalmente el de McCoy (27), que muestra que cuando estas barreras se modifican favorablemente para el paciente oncológico, el pronóstico de su enfermedad mejora.

En cuanto al actual estudio y como se aprecia por los resultados, al Instituto Nacional de Cancerología de Bogotá acuden muchos pacientes en condiciones de pobreza y vulnerabilidad de diversas regiones del país, los cuales son potenciales usuarios de un programa de apoyo en alojamiento y manutención mientras reciben tratamiento. Con este fin existen unos programas de albergue que el Instituto ayuda a financiar, pero que resultan totalmente insuficientes para las necesidades, por lo cual, es conveniente buscar otras alternativas de alojamiento y soporte a la permanencia en la ciudad de Bogotá, para obtener costos más razonables y mejores condiciones, que faciliten el acceso al tratamiento especializado.

Al no existir esta alternativas, los costos para acceder al mismo se incrementen notoriamente y por lo tanto es de suponer que un gran número de pacientes decide no desplazarse a la ciudad de Bogotá, sino limitarse a los tratamientos que se puedan ofrecer a nivel regional, de tal manera que esta limitación puede estar influyendo notablemente en el aumento del índice de mortalidad por cáncer, debido a las barreras ocultas de acceso al tratamiento. Los aspectos analizados nos confirman la necesidad de plantear alternativas de solución; En este sentido, un primer escenario que resulta indispensable en el corto plazo, es reforzar y mejorar la alternativa de los albergues actuales y de las casa de familia que acogen a enfermos con cáncer, para tal fin se puede generar una red de apoyo, vinculando diversas organizaciones. Por ejemplo, es posible que las Universidades que tienen convenio docencia servicio con el Instituto ayuden en la preparación y el cuidado de los cuidadores de estos enfermos, a lo que se puede sumar un convenio con una empresa de transporte, en el cual y con subsidio del Estado se movilicen en vehículos, debidamente acondicionados, los pacientes con cáncer que acuden a la ciudad, En cuanto a mejorar la actual capacidad de los albergues el principal reto es de tipo financiero, pero si el costo se dispersa entre todas las entidades directamente responsables del tratamiento de esta patología, esto no seria muy gravoso para ninguna, ya que por ejemplo con un incremento de \$ 6 por cada \$ 1000 de la actual facturación de servicios, la capacidad actual de los albergues se podría casi duplicar. 
Igualmente y en el mediano plazo y dado que hay un proyecto regional denominado Ciudad-Salud que pretende posicionar esta zona de la ciudad como un lugar de excelencia en la prestación de estos servicios, de tal forma que sea centro de referencia Nacional e Internacional, es posible pensar en un gran centro de alojamiento para familiares y pacientes, con todas las condiciones de infraestructura y confort. Este no sólo vendería servicios a personas con capacidad de pago, sino que con parte de las utilidades y con cofinanciación por parte del Estado, los aseguradores, los prestadores y posiblemente diversas organizaciones de utilidad común, serviría para acoger a población vulnerable que requiere de la atención. Finalmente, debe fortalecerse el proyecto de Sistema Nacional de Servicios Oncológicos, Red Nacional de Cáncer (28), con el cual es posible mejorar la capacidad resolutiva de las regiones y concentrar los esfuerzos del INC en los casos más complejos •

Agradecimientos. A Carlos Vicente Rada Escobar, Director General del Instituto Nacional de Cancerología E.S.E., quién brindó apoyo incondicional para la recolección de información y demás actividades relacionadas con el desarrollo del trabajo.

\section{REFERENCIAS}

1. República de Colombia. LEY 789 de 2002. Por la cual se dictan normas para apoyar el empleo y ampliar la protección Social y se modifican artículos del código sustantivo del trabajo. Diario oficial $N^{\circ} 45.046$ de 27 de diciembre de 2002.

2. Torres M. Reseña del libro Derecho a la Salud en Colombia de Paredes N. Bogotá, GTZ y CINEP. Revista Gerencia y Políticas de Salud. Dic 2003; 2(5): 119.

3. Bases del Plan Nacional de Desarrollo 2002-2006. Hacia un Estado Comunitario. Presidencia de la República-Departamento Nacional de Planeación. Bogotá: Departamento Nacional de Planeación; 2002.

4. Wiesner C, Tovar S, Díaz M, Pardo C. El cáncer - Aspectos básicos sobre su prevención, diagnóstico y tratamiento. Ministerio de la Protección Social - Instituto Nacional de Cancerología E.S.E.; 2004.

5. Plazas M, Ospino R, Mejía A. Evaluación de los Servicios de Radioterapia en Colombia. Instituto Nacional de Cancerología, Ministerio de la Protección Social, Organización Panamericana de la Salud, Organización Mundial de la Salud. Bogotá; 2005.

6. Vélez, A. La acción de tutela: ¿un mecanismo de protección del derecho a la salud y un proceso alterno para acceder a servicios de salud? Revista Colombia Médica. 2005; 36(3): 199-208.

7. Jaramillo I. Evaluación de la descentralización de la salud y la reforma de la Seguridad Social en Colombia. Gac Sanit. 2002; 16(1):48-53.

8. Hernández M. Reforma sanitaria, equidad y derecho a la salud en Colombia. Cad. Saúde Pública. 2002; 18 (4): 991-1001.

9. Agudelo C. Sistema de salud y plan de desarrollo: Cobertura y equidad. Rev. Salud Pública (Bogotá). 1999; 1(2):117-127.

10. Eslava JC. Seguridad social en salud: Reforma a la reforma. Rev. Salud Pública (Bogotá) 1999; 1(2):105-116.

11. Céspedes JE, Jaramillo I, Martínez R. Efectos de la reforma de la seguridad social en salud en Colombia sobre la equidad en el acceso y la utilización de servicios de salud. 
Rev.Salud Pública (Bogotá). 2000; 2(2):145-164.

12. Hernández M. El derecho a la salud en Colombia: Obstáculos estructurales para su realización. Rev. Salud Pública (Bogotá). 2000; 2(2):121-144.

13. Málaga $\mathrm{H}$, Latorre $M$, Cárdenas J. Equidad y reforma en salud en Colombia. Rev. Salud Pública 2000; 2(3):193-219.

14. Restrepo M. 2000. Elementos para una doctrina del derecho a la salud en Colombia. In: Giraldo JC. Ejercicios del Derecho a la Salud en Colombia. Bogotá: Defensoría del Pueblo. pp. 51-121.

15. Regidor E, Martínez D, Astasio P. Asociación de los ingresos económicos con la utilización y la accesibilidad de los servicios sanitarios en España al inicio del siglo XXI. Gac Sanit. 2006; 20 (5): 352-359.

16. Drever F, Whitehead M. (eds.). Health inequalities. Decennial supplement. London: Stationery Office; 1997.

17. Mackenbach JP, Bos V, Andersen $\mathrm{O}$. Widening socioeconomic inequalities in mortality in six European countries. Int J Epidemiol. 2003;32:830-7.

18. Kunst AE, Bos V, Lahelma $E$. Trends in socio-economic inequalities in self assessed health in ten European countries. Int J Epidemiol. 2005;34:295-305.

19. Mejia-Mejia A, Sanchez-Gandur A, Tamayo-Ramirez J. Equidad en el Acceso a Servicios de Salud en Antioquia, Colombia. Rev. salud pública (Bogotá). 2007; 9(1): 26-38.

20. Ruiz-Rodriguez M, Lopez-Moreno S, Avila-Burgos L. Utilización de servicios médicos por una población desplazada en Bucaramanga, Colombia. Rev. salud pública (Bogotá). 2006; 8(3):197-206.

21. Modeste N, Caleb-Drayton V, Montgomery S. Barriers to early detection of breast cancer among women in a Caribbean population. Rev Panam Salud Publica, Mar. 1999; 5(3): 152-156.

22. Angus J, Paszat L, McKeever, P. Pathways to breast cancer diagnosis and treatment: exploring the social relations of diagnostic delay. Texto contexto-enferm. 2007; 16 (4): 591-8.

23. Fonseca S, Gutiérrez M, Adami N. Avaliação da satisfação de pacientes oncológicos com atendimento recebido durante o tratamento antineoplásico ambulatorial. Rev. bras. enferm. sep./oct. 2006; 59(5): 656-660.

24. Restrepo J, Mejía A, Valencia M. Accesibilidad a la citología cervical en Medellín, Colombia en 2006. Rev. Esp. Salud Publica, Nov-Dic. 2007; 81(6): 657-666.

25. Palacio-Mejía, L, Rangel-Gómez G, Hernández-Ávila M. Cervical cancer, a disease of poverty: mortality differences between urban and rural areas in Mexico. Salud pública Méx, 2003; 45 (supl.3): 315-325.

26. Lucumi D, Gómez L. Accesibilidad a los servicios de salud en la práctica de citología reciente de cuello uterino en una zona urbana de Colombia. Rev. Esp. Salud Pública, mayo-jun. 2004; 78 (3): 367-377.

27. McCoy C, Pereyra M, Metsch L. A community-based breast cancer screening program for medically underserved women: its effect on disease stage at diagnosis and on hazard of death . Rev Panam Salud Publica, Mar. 2004; 15(3): 160-167.

28. INC 2008. Bases conceptuales y operativas de la Red Nacional de Cáncer [Internet]. Disponible en: http://www.incancerologia.gov.co/paginas.aspx?cat_id=0\&pub_id=236. Consultado: Agosto 2008. 